\title{
Mongili Alessandro \& Pellegrino Giuseppina (eds) (2014) Information Infrastructure(s): Boundaries, Ecologies, Multiplicity. Newcastle upon Tyne: Cambridge Scholars Publishing.
}

\author{
Jean-Christophe Plantin \\ j.plantin1@lse.ac.uk
}

The book Information Infrastructure(s): Boundaries, Ecologies, Multiplicity contains fourteen chapters presenting a variety of research on information infrastructures. The book is organized in four parts. The first part entitled "Designing and articulating information infrastructures" presents three studies promoting concepts that reflect the tensions inherent to infrastructure development. Mongili goes beyond the separation between users and designers in creative practices by showing how software developers and designers are always users of the infrastructural capacities and the technical environment they work in. Pellegrino presents the central concept of ductility to characterize contingency in information infrastructures, and by contrasting it to resilience, he shows how boundary objects are always enacted through a mixture of flexibility and consistency. Klein and Schellhammer use the introduction of a new automatic drug dispensing to highlight how existing legislation and industrial processes can prevail and hinder innovative practices.

The second part, "Information infrastructures as ecological tools," consists of three chapters that apply Bowker and Star's methods of infrastructure as ecology of practices, and show the constant adjustments between the different components of infrastructures. Poderi uses such ecological approach to ethnographically study the multiplicity of users' contributions in open source video game; Neresini and Viteritti analyse laboratory kits, these ready-made substances or material for procedures, and show how they are simultaneously fixed and flexible to use and interpret. Still in the laboratory environment, Crabu shows the active role of protocols in shaping infrastructures.

The third part, "Users, information infrastructures and mobilities," comprises four chapters. The first, from Denis and Pontille, addresses the tension between users and established contributors in a participatory geographic database, with opposite foci on visual rendering of the map vs. data coherence; Isabella shows, with the case study of a call center, how the category of "user" is constantly moving between managerial attempts to standardize work procedure and technicians' desire to keep some autonomy on their work. Additionally, Lazzer and Giardullo analyze online networks of actors related to online publishing (ebooks), and Mitrea discusses the concept of 'dispositif' in relation to scripts and programs of action, to think about shifts in mobilities in modern and postmodern societies.

The fourth and last part, 'On boundary objects, and on multiplicity, presents four case studies (a spin-off company, by Miele; the threshold for advanced maternal age, by Turrini; science parks, by Cozza; ICT convergence, by Lugano), each presenting the relevance of boundary object as a concept to think about the management of innovation and the study of organizations.

It is remarkable that this book makes an equal theoretical and empirical contribution to the study of infrastructure, without prioritizing one over the 
other. All the fourteen contributions of this book are based on a rich variety of case studies, and if the breadth of results and the degree of involvement towards the case studies are different, they all present a strong commitment to the empirical analysis of information infrastructures. The reader will certainly enjoy the possibility of learning at the same time about such a variety of topics as automatic drug dispensing in Germany, science parks in Italy, or participatory mapping initiatives in France. But the book also shows a great richness in its theoretical contributions: through the various case studies, the authors interrogate and adapt building block concepts of infrastructure studies, with an emphasis on boundary objects, ecology, users, and design. The introduction of the book similarly constitutes a very strong summary of the history and current research in the study of information infrastructures, as well as the specificity of the research network on STS in Italy.

The accumulation of different contributions is particularly relevant concerning the notion of boundary objects. It takes part in lively debates in the field on the relevance and domains of applications of the concept (previously debated in the special issue on boundary object in La revue des connaissances in 2009 (volume 3, issue 1), and more recently with the book Boundary Objects and Beyond (Bowker et al., 2016). It makes a compelling case to consider situations where the introduction of a boundary object does not result in the expected stabilisation: on the contrary, various contributions show how boundary objects can become an obstacle to cooperation. Finally, the different contributions of this book show the relevance of the concept beyond STS, principally in organization and management studies.

The part IV on boundary object is very coherent: however, it is relatively harder to see the contributions made concerning the concepts of ecology and multiplicity, as they are not clearly defined in the introduction, and the reader is left wondering about the specificity of these concepts across all the different contributions. In the same vein, the four parts chosen to arrange the chapters of the book are not the most convincing: it is hard to see the coherence between them, and topics are frequently treated across several parts (such as the topic of "users"). Additionally, some editing work would have prevented the repetition in several chapters of similar theoretical parts (such as the literature review on the concept of infrastructure, the relevance of Bowker and Star...), as it is not always clear how they differ across authors.

If a strength of the book is to articulate empirical and theoretical contributions to show the variety of the STS toolbox, there is a tendency among some authors to simply present their research object through the grid of the STS vocabulary: for some chapters, knowing whether or not the object investigated constitutes a boundary object, or an infrastructure, seems to be the most important contribution, when this constitutes more a start than a result. Similarly, there is in several chapters the tendency to interchangeably use a variety of STS/infrastructure studies concepts for the same object (boundary object, program of action, script, etc.), without clearly identifying the difference between them. Finally, some authors state that their main contribution is to reveal the adaptability of device/processes that are supposedly rigid. It is coherent with the nature of boundary object, but this is hardly an innovative result.

More generally, it is unclear whether his book summarizes past research, or draws a roadmap of future research in the field. It is made clear that it is based on past events and panels, and the variety of chapters makes a strong case to show the vitality of the field of infrastructure studies. However, it is unsure if the reader can close this book and have a clear view of where the study of information infrastructures is going in a near future. Recent panels in STS conferences have raised the question of the next steps for infrastructure studies, potentially even beyond the term ("Beyond Infrastructure: Theorizing Alternatives and Absences," 4S 2014). Relatedly, the concept is now competing with others, such as "platforms" ("Cage fight: infrastructure studies vs. platform studies," 4S 2015), which calls for a closer interrogation of the specificity of infrastructure studies in the digital age. This book sadly does not engage with such questions, and does not to draw a prognostic of the future of the field.

Despite these limitations, this edited book is a great contribution to the study of information infrastructures, and shows the variety and vitality 
of this field of inquiry. The reader will appreciate the feeling of intellectual adventure that goes through this book, with the touching foreword by Geof Bowker or the introduction as an homage to the contribution of Leigh Star. Most importantly for the field, it shows the vitality of scholarship that does not come from an English speaking country, reflecting current efforts in STS to connect with larger researchers' communities.

\section{References}

Bowker G C, Timmermans S, Clarke A, \& Balka E (eds) (2016) Boundary Objects and Beyond: Working with Leigh Star. Cambridge, Massachusetts : The MIT Press.

Revue d'anthropologie des connaissances (2010) 4(1) 